\title{
Utilidad del índice glucémico y la carga glucémica de los alimentos como herramientas de recomendación dietética
}

\author{
Usefulness of glycemic index and glycemic load of foods as dietary recommendation tools \\ José Antonio Estefes-Duarte ${ }^{a}$, Uriel González-Lemus ${ }^{a}$, Alma Delia Hernández-Fuentes ${ }^{a}$, \\ Anuar Jottar-Bernal ${ }^{a}$ y Rafael Germán Campos-Montiel ${ }^{a^{*}}$
}

\begin{abstract}
:
Carbohydrates are a wide family of molecules that form an important part of the diet and represent optimal substrates for obtaining energy. For some years, carbohydrates have been investigated for possible associations between high consumption patterns and the occurrence of some chronic pathologies. In the 80 's, a concept trying to explain the behavior of different types of food carbohydrates on plasma glucose levels, was developed. Since its inception to date, the relevance of this concept, known as glycemic index, has been subject of controversy among nutrition professionals and the scientific community for different reasons. More recently, another concept (the glycemic load) has attempted to better explain the impact of carbohydrates on the blood, however, their real importance and usefulness in dietetics are still under debate. This document offers an overview of the origin and usefulness of these concepts, as well as a compilation of scientific evidence that relates these concepts to development or treatment of some physiological and pathological situations.
\end{abstract}

Keywords:

Carbohydrates, glycemic index, glycemic load, chronic disorders

\section{Resumen:}

Los carbohidratos son una amplia familia de moléculas que forman parte importante de la dieta y representan sustratos óptimos para la obtención de energía. Desde hace algunos años los carbohidratos han sido investigados por la posible asociación de su consumo elevado con el desarrollo de patologías crónicas. En la década de los 80’s surgió un concepto que intentaba explicar el comportamiento de los diferentes tipos de carbohidratos de los alimentos sobre los niveles de glucosa plasmática. La utilidad de este concepto, conocido como índice glucémico desde su creación hasta la fecha ha sido objeto de controversia entre los profesionales de la nutrición y la comunidad científica debido a diferentes razones. Más recientemente, otro concepto (la carga glucémica), ha intentado explicar de una mejor manera el impacto de los carbohidratos en la glucemia, no obstante, su importancia y utilidad reales en la dietética aún se encuentran en debate. Este documento ofrece un panorama general en torno al origen y utilidad de estos conceptos, así como una recopilación de evidencia científica que relaciona estos conceptos con el desarrollo, o bien, con el tratamiento de algunas situaciones fisiológicas y patológicas.

Palabras Clave:

Carbohidratos, índice glucémico, carga glucémica, enfermedades crónicas

\section{Introducción}

En los últimos años, una creciente toma de conciencia por parte de los consumidores sobre el contenido nutrimental de los alimentos [1], aunada a la preocupación de la población por problemas de salud cada vez más comunes como la diabetes, obesidad, enfermedad coronaria, cáncer, entre

a'Universidad Autónoma del Estado de Hidalgo. José Antonio Estefes-Duarte, https://orcid.org/0000-0001-6824-0033, Email: jose_estefes@uaeh.edu.mx; Uriel González-Lemus, https://orcid.org/0000-0002-7050-0874, Email: uriel_gonzalez@uaeh.edu.mx; Alma Delia Hernández-Fuentes, https://orcid.org/0000-0003-2592-6689, Email: almah@uaeh.edu.mx; Anuar Jottar-Bernal, https://orcid.org/00000003-2997-9219, Email: jo133673@uaeh.edu.mx; Rafael Germán Campos-Montiel, https://orcid.org/0000-0001-7382-5538, Email: rcampos@uaeh.edu.mx

*Autor de Correspondencia: rcampos@uaeh.edu.mx 
otros, han impulsado el interés científico para estudiar las condiciones de alimentación del ser humano que pudieran estar influyendo en el desarrollo de dichas patologías [2]. Un grupo de nutrimentos que ha despertado especial interés es el de los carbohidratos, ya que su consumo excesivo se ha relacionado con problemas de salud como la diabetes y el cáncer [3]. De hecho, en la última década, el escrutinio en torno a los carbohidratos ha comenzado a aproximarse al de las grasas [4], a pesar de que los hidratos de carbono son la principal fuente de energía de los seres humanos y cubren aproximadamente el $60 \%$ del aporte energético total [5]. Actualmente existen dos herramientas empleadas comúnmente para conocer el efecto de los carbohidratos de los alimentos en la glucosa plasmática: el índice glicémico (IG) y la carga glucémica (CG), sin embargo, el uso de estas herramientas dietéticas hasta hoy en día es controversial y debatido [6]. Por ello, el objetivo de este trabajo es analizar las consideraciones e implicaciones en torno al uso del índice glucémico (IG) y la carga glucémica (CG) como herramientas de recomendación dietética, en relación a la situación fisiológica o patológica del individuo. Además, se ofrece una opinión basada en la evidencia y una postura personal de enfoque clínico en este documento.

\section{Carbohidratos}

Los carbohidratos, hidratos de carbono o glúcidos son aldehídos, polihidroxicetonas, alcoholes, ácidos, sus derivados simples y sus polímeros que se unen mediante enlaces tipo acetal [7]. Son fuentes óptimas de sustratos necesarios para que el organismo los convierta en energía en forma de ATP (trifosfato de adenosina) [8]. Químicamente, se pueden clasificar de acuerdo con el número de unidades monoméricas repetitivas que forman una cadena correspondiente a un carbohidrato cualquiera (grado de polimerización) y básicamente se distinguen tres grupos principales, a saber: azúcares (monosacáridos como glucosa, fructosa y galactosa; disacáridos como lactosa y polioles), oligosacáridos (como rafinosa y estaquiosa) y polisacáridos (como almidón, celulosa, hemicelulosa y pectina) [7].

\section{1 ¿Carbohidratos simples y complejos?}

Aunque en la dietética tradicional los carbohidratos se han clasificado como "simples" o "complejos" basándose en la cantidad de monosacáridos 0 disacáridos presentes en cada molécula [9], realmente el término "carbohidratos complejos", (que apareció por primera vez en 1977 en un informe llamado "Objetivos Alimentarios para los Estados Unidos") [10], originalmente se refería únicamente a frutas, verduras y granos enteros y se acuñó en gran medida para distinguir a los azúcares (azúcares simples) de otros hidratos de carbono [7], por lo cual su validez actual parece haber disminuido, ya que en ciertos carbohidratos, el término "complejos" carece de su enfoque original. De modo que resulta más conveniente analizarlos mediante la utilización de sus nombres químicos correspondientes.

En general, los carbohidratos presentes en los alimentos, desde los almidones hasta el azúcar de mesa, comparten por lo menos una propiedad biológica básica: pueden ser transformados en glucosa mediante la digestión [11]; sin embargo, existe una percepción global de que los "carbohidratos complejos" ejercen menor efecto en la glucosa sanguínea que los carbohidratos simples, lo cual no en todos los casos es verdad. De hecho, ahora se sabe que algunos carbohidratos complejos tienen mayor impacto en la glucosa sanguínea que ciertos carbohidratos clasificados como simples [9]. Como este y otros aspectos originaron la necesidad de explicar con mayor veracidad el comportamiento de la glucosa sanguínea tras la ingestión de carbohidratos de distinta naturaleza.

\subsection{Origen de los términos índice glucémico y carga glucémica}

Si bien es cierto que alrededor del mundo se conoce que la dieta habitual es el mayor factor de riesgo modificable para la prevención de enfermedades crónico-degenerativas como diabetes [12], obesidad, enfermedades cardiovasculares, cáncer etc., y se han estudiado los cambios fisiológicos que surgen de la modificación de la calidad y cantidad de ciertos nutrimentos como las grasas, el papel de los carbohidratos actualmente parece más polémico [13]. Por ejemplo, en algún momento se pudo observar que, diferencias tanto en la cantidad como el tipo de carbohidratos 
consumidos en la dieta, ejercían un efecto distinto sobre la secreción de insulina y la glicemia postprandial [14], y que dichas diferencias no podían ser explicadas por la longitud de la cadena de los carbohidratos (i.e. carbohidratos simples y complejos) [15]. Fue entonces que surgió el índice glicémico (IG) con la finalidad de describir el grado en el que los diferentes alimentos, principalmente aquellos que contienen cantidades importantes de carbohidratos, provocan cambios en la glucosa postprandial de los individuos [16]. De este concepto posteriormente derivaría un nuevo término conocido como "carga glicémica" (CG) [1]; no obstante, el fundamento de estos dos términos se basa en algo conocido como "respuesta glucémica". En ayuno, la concentración plasmática de glucosa tiene un valor dado que se conoce como basal; al consumir un alimento que contiene carbohidratos, esta concentración plasmática sube a un nivel máximo y posteriormente baja hasta ubicarse en su valor de origen. Esto es lo que se denomina respuesta glucémica [2].

Tabla 1. Ventajas y desventajas del uso del índice glicémico como herramienta de valoración de la calidad de un alimento

\section{Ventajas}

Medida sencilla, no es necesario grandes equipamientos ni reactivos para llevarla a cabo

Existe mucha información al respecto

\section{Desventajas}

En la descripción de los alimentos a evaluar no se hace referencia a ninguna propiedad cuantitativa, ni química ni física, salvo el contenido de hidratos de carbono disponibles

Todo alimento evaluado debe tener la misma cantidad de hidratos de carbono disponible

No existe un alimento perfectamente estandarizado (cualitativamente), por lo que los resultados pueden variar según como esté preparado (pan blanco principalmente)

\section{Consecuencia}

Se puede realizar fácilmente a cualquier persona, con cualquier alimento, con poco presupuesto

Existen artículos en revistas especializadas que resumen valores de IG de un gran número de alimentos por lo que se puede tener una guía fácil al respecto

\section{Consecuencia}

Alimentos con un mismo contenido y tipo de hidratos de carbono pueden dar IG distintos, siendo imposible explicar la razón de esto. No existe ninguna relación cuantitativa entre propiedades del alimento y su IG. Reproducibilidad de cada experimento muy limitada.

La interpretación de los resultados muchas veces es incorrecta ya que no se consideran los tamaños de las porciones a consumir; esto entrega resultados que en algunos casos son poco extrapolables a un alimento real

La comparación entre distintos experimentos es casi imposible

\section{1 Índice glucémico}

El índice glucémico, entonces, es una medición in vivo que considera la compleja interacción de 
factores que determinan la respuesta glucémica [17]. El término IG fue concebido y comunicado en el año de 1981 por David Jenkins y colaboradores en la Universidad de Toronto Canadá [4], como una herramienta para el manejo dietético de la diabetes mellitus tipo 1 [18], y se basó en la hipótesis de que los carbohidratos simples como la glucosa, el azúcar de mesa y la maltosa, inducían un incremento de la glucemia más rápido y mayor que los "carbohidratos complejos" como el almidón, hecho que sería cuestionado posteriormente y quizá uno de los motivos por los cuales su utilización como herramienta de control glucémico y prevención de enfermedades crónicas es controversial desde hace aproximadamente 20 años.

La actual medición del IG compara los efectos en la elevación de la glucosa sanguínea después de ingerir 25 [12] o $50 \mathrm{~g}$ de un alimento estándar (usualmente glucosa o pan blanco) con los efectos de ingerir 25 o 50 gramos de carbohidratos disponibles de un alimento en cuestión [4]. De esta medición se obtiene un gráfico que describe el ascenso y descenso de la glucosa, el cual es medido como el área bajo la curva obtenida después de 2 horas de la ingestión del alimento [16]. Entonces se divide el área bajo la curva del alimento en cuestión por el área bajo la curva del alimento estándar al cual se le asigna un valor de $100[4,8]$.

El cociente resultante (IG), entonces, es una comparación entre la glucosa de cualquier alimento y la glucosa del estándar. Si al alimento en cuestión se le calcula un valor menor al 100 , se espera que ese alimento impacte la glucosa sanguínea en menor grado que la glucosa; por el contrario, si al alimento analizado se le otorga un valor mayor a 100 , teóricamente ese alimento impactará la glucosa sanguínea del consumidor en mayor medida que la glucosa. De acuerdo con esto, los valores actuales del IG se agrupan en tres categorías. IG alto $\geq 70$, IG intermedio 56-69, IG bajo de 0-55 [18].

Distintas implicaciones inherentes al cálculo del IG proporcionan, según los profesionales, distintas ventajas y desventajas a la hora de evaluar la calidad de un alimento. Algunos de estos aspectos se resumen en la Tabla 1.
En 1997, algunos años después de la creación del concepto de IG, un grupo de investigadores de la Universidad de Harvard, [19] definieron un nuevo concepto; el de la carga glicémica (CG), que cuantificaba el impacto de una porción habitual de un alimento con determinado IG (18); es decir, la CG toma en cuenta no solo la rapidez de un alimento en convertirse en azúcar en la sangre, sino también la cantidad de carbohidratos que tiene ese alimento en particular para poder determinar el grado de absorción de la glucosa $(1,8,18)$. Por lo que, si se desea conocer el impacto en sangre de un alimento individual, la carga glucémica podría ser más relevante que el índice glicémico.

Su estimación puede hacerse según la siguiente fórmula: $C G=I G \times$ contenido neto de hidratos de carbono por porción en g/100 [18]. En este sentido, se consideran alimentos con CG alta si presentan un valor de 20 o más. Si la carga va de 11 a 19 es considerada media y los valores de CG por debajo de 10 son bajos [1, 8,18,20].

Es necesario entender que, aunque la $C G$ es una medida derivada del IG, ambas mediciones no tienen necesariamente una correlación directa $[2,20]$; esto quiere decir que algunos alimentos con un IG alto como las zanahorias pueden tener una CG baja [4], todo dependerá del contenido neto de carbohidratos [2]. De tal manera que cuando se ingiere la misma cantidad (en gramos) de dos alimentos con IG similar, el aumento del nivel de glucosa en sangre será menor con el alimento que tenga un menor contenido de carbohidratos. De igual manera, si se comen dos alimentos con igual IG, pero con porciones distintas, la menor porción producirá un menor aumento de la glucosa [11, 21,22,23]. En la Tabla 2 se resume el índice glucémico y la carga glucémica de algunos alimentos.

\subsubsection{Carga glucémica}




\section{Tabla 2}

Ejemplos de índice glucémico y carga glucémica de algunos alimentos comunes

\begin{tabular}{lcccc}
\hline Alimento & IG & TP & CD & CG \\
\hline Melón & 72 & 120 & 6 & 4 \\
Helado (alto en & 37 & 50 & 9 & 4 \\
grasa) & 74 & 150 & 20 & 15 \\
Puré de papa & 47 & 180 & 48 & 23 \\
Macarrones & 64 & 150 & 36 & 23 \\
Arroz precocido & 65 & 60 & 40 & 26 \\
Barra de chocolate & 65 & 22 & 13 \\
Hojuelas de avena & 58 & 250 & 26 & 21 \\
Hojuelas de maíz & 81 & 30 & 26
\end{tabular}

Tomado de las tablas internacionales de BrandMiller [14]. IG: Índice glucémico, TP: Tamaño de la porción (g), CD: Carbohidratos disponibles (g), CG: Carga glucémica.

\subsection{Factores que afectan el índice glucémico y la carga glucémica}

\subsubsection{Factores propios del alimento}

El IG de un alimento está influenciado por diferentes factores que además del tipo de hidratos de carbono influyen decisivamente en el comportamiento glucémico de un alimento. En este sentido, se debe tener en cuenta que desde la ingestión del alimento, hasta que finalmente pasa a la sangre y eleva la glucemia, representa un periodo largo y complejo donde intervienen diferentes factores tanto físicos y químicos que interactúan en el alimento entre los que destacan las técnicas de procesamiento (e. g. molienda) [24], técnicas culinarias (calor aplicado, cantidad de agua y tiempo de preparación), tipo de almidón (relación amilosa-amilopectina), contenido de fibra, tipo de hidratos de carbono [12], contenido de grasas o proteínas ya que las proteínas están constituidas de unidades denominadas aminoácidos, que forman largas cadenas. Debido a la compleja naturaleza molecular de las proteínas, el organismo necesita más tiempo para disociarlas. Por otra parte, la acidez por la utilización de ingredientes como el vinagre [18], pueden modificar la estructura y el contenido de almidón disponible, lo que se ve reflejado en la disminución del IG. o por los niveles naturales de ácidos orgánicos [24] que retrasan el vaciado gástrico (ya que aumentan la concentración de almidón resistente) y reducen el índice glucémico [25]. Por ejemplo, la estructura física de un alimento es un aspecto crucial que modifica el IG. Alimentos compactos como las semillas, ciertos vegetales y las leguminosas, impiden la penetración de las enzimas digestivas de almidón, las amilasas. Esto se traduce en menor glucosa liberada en el torrente sanguíneo y por ende un menor IG. Tales alimentos son descritos algunas veces como almidones de glucosa lentamente disponible. En contraste, alimentos porosos como el pan, permiten rápidamente la penetración enzimática. El resultado es un elevado IG debido a la rápida digestión y rápida liberación de glucosa en el torrente sanguíneo. Los alimentos de esta categoría son referidos algunas veces como almidones de glucosa rápidamente disponible [4].

En lo que respecta al tipo de azúcar presente en el alimento, la glucosa, por ejemplo, tiene un elevado IG mientas que la fructosa lo tiene bajo. La sacarosa y el jarabe de maíz alto en fructosa poseen aproximadamente $50 \%$ de glucosa y $50 \%$ de fructosa, así que sus IGs correspondientes reflejan la mezcla de esos dos monosacáridos lo que los vuelve en realidad alimentos con IG moderado [4]. La presencia de grasas o proteína en el alimento también puede modificar las tasas de digestión y absorción del almidón y, por lo tanto, modificar su IG. Para el caso del contenido de fibra, se ha aceptado que los alimentos ricos en fibra pueden retrasar la absorción de la glucosa en el intestino delgado debido a que la fibra puede aumentar la viscosidad del bolo alimenticio en el intestino delgado interfiriendo en la absorción de macronutrientes, influyendo así en las concentraciones de glucosa postprandial en sangre, sin embargo, se ha sugerido que el efecto de la fibra dietética en el control de la glucosa sanguínea podría ser demasiado pequeño como para ser clínicamente relevante [26]. Por tal motivo, el IG de un alimento no puede predecirse por su contenido de fibra $u$ otros nutrimentos, o por considerarlo un "producto integral" o "cereal integral", para los cuales, dicho sea de paso, no hay definiciones universalmente aceptadas [20]. Algunos factores de los alimentos que influyen en las propiedades glucémicas de los alimentos se encuentran resumidos en la Tabla 3. 
Tabla 3

Factores propios de los alimentos que influyen en la glucemia plasmática

\begin{tabular}{ll}
\hline Factor & Ejemplo \\
\hline Cantidad de carbohidratos & \\
Naturaleza de los monosacáridos & Glucosa \\
& Fructosa \\
& Galactosa \\
Naturaleza del almidón & Amilosa \\
& Amilopectina \\
& Interacciones almidón- \\
& nutrientes \\
& Almidón resistente \\
Preparación y procesamiento del alimento \\
& Grado de gelatinización \\
& del almidón \\
& Tamaño de partícula \\
& Forma del alimento \\
& Estructura celular \\
Otros componentes de los alimentos \\
Grasa y proteína \\
Fibra dietética \\
Antinutrientes \\
Ácidos Orgánicos \\
\hline
\end{tabular}

Franco-Mijares et al [8]

\subsubsection{Factores propios del consumidor}

Además de las características del alimento, también intervienen condiciones propias de cada sujeto que alteran su respuesta glucémica. El estado fisiológico en el que se encuentra en el momento del consumo determina la ruta metabólica que seguirán los nutrimentos y cómo se reflejarán en la glucemia [27]. Es decir, la respuesta metabólica tras la ingestión de un alimento se vuelve variable en función de las condiciones fisiológicas y patológicas de cada individuo (e. g. la respuesta metabólica de pacientes diabéticos tras la ingestión de carbohidratos influye en el efecto glucémico de los alimentos). En la Tabla 4 se muestran algunos factores externos a los alimentos que afectan la respuesta glucémica y por tanto el IG y CG de los alimentos.

\subsection{Problemas en la medición del índice y la carga glucémica}

La utilidad del IG y la CG ha sido cuestionada en muchas ocasiones. Existen evidencias que el IG de un alimento difiere cuando se mide en forma aislada o en el contexto de una comida mixta, forma esta última, como el ser humano consume habitualmente los alimentos [18]. Fallas al considerar la respuesta insulinémica, el alto grado de variabilidad intra-einter sujetos y una pérdida de poder discriminatorio cuando los alimentos se combinan en platillos preparados son algunos de los argumentos que se consideran a la hora de evaluar la confiabilidad de estas herramientas. Además, alimentos con un alto contenido de azúcar (sacarosa) y aquellos que contienen tanto grasas como carbohidratos pueden tener un bajo IG, pero no pueden ser considerados elecciones apropiadas principalmente por su alta densidad energética y el tipo de grasas [20]. La falta de estandarización de la técnica de medición y el uso de diferentes estándares de referencia y porque en ocasiones la aplicación de este concepto lleva a recomendaciones nutricionales desequilibradas [18] son algunas otras consideraciones que se discuten en torno a esto (Tabla 4).

Por otra parte, algunos problemas en la medición particularmente de la CG pudieran atribuirse a que la CG es directamente proporcional a la cantidad de alimentos consumidos, y, contrario a toda lógica, el área bajo la curva $(A B C)$ de la glucosa en sangre no aumenta en proporción directa a la cantidad de alimento consumida. Por ejemplo, [20] se reportó que comer seis veces una porción de pan resulta en un incremento de aproximadamente tres veces en el $A B C$. Esto quiere decir que, entre mayor es la cantidad de alimento que se consume, la tasa de incremento del $A B C$ decrece, probablemente debido a complejos ajustes hormonales que el organismo realiza tras detectar grandes cantidades de alimento. Además, las críticas en cuanto a la medición de la CG apuntan a que ésta última toma como base una medida que por sí sola ya es imprecisa, el IG, lo cual amplifica el error potencialmente al multiplicarlo por los gramos de carbohidratos [4].

\subsection{Publicaciones y resultados controversiales}

Diversos estudios han demostrado el efecto positivo de las dietas con bajo IG sobre los lípidos en 
pacientes hiperlipidémicos y diabéticos, aunque su recomendación para uso en la clínica ha sido controvertida. Por ejemplo, el comité de expertos en nutrición de la OMS y la FAO recomendó que el IG se deba considerar al comparar alimentos dentro del mismo grupo. Asimismo, el grupo de nutrición de la Asociación Europea para el Estudio de Diabetes (EASD por sus siglas en inglés) señala que los alimentos con bajo IG deben sustituir a los alimentos con alto IG. Sin embargo, la Asociación Americana de Diabetes (ADA) omite proponer la utilización del IG en el tratamiento nutricional $[28,29]$.

\subsection{1 Índice y carga glucémica en diabetes}

Gran cantidad de estudios han sido publicados en base a la investigación de los efectos de dietas con bajos IG y $C G$ en el control específico de la diabetes. Un meta-análisis de 14 estudios que involucraban 356 sujetos con diabetes tipo 1 y 2 encontró que dietas con bajo IG pueden mejorar el control glucémico en una medida similar a la observada con los medicamentos hipoglucemiantes post-alimentos [21].

\section{Tabla 4}

Factores externos a los alimentos que afectan la respuesta glucémica y su estudio

\begin{tabular}{|c|c|c|c|}
\hline & Factor & Ejemplo & Respuesta glucémica \\
\hline \multirow[t]{2}{*}{ Humano } & Estado de salud & $\begin{array}{l}\text { Diabetes, insulino- } \\
\text { sensibilidad y } \\
\text { tolerancia a la glucosa }\end{array}$ & $\begin{array}{l}\text { Disminuye la precisión, } \\
\text { sólo si existe variabilidad } \\
\text { inter-individual }\end{array}$ \\
\hline & $\begin{array}{l}\text { Estado de } \\
\text { entrenamiento }\end{array}$ & $\begin{array}{l}\text { Estudios sin control de } \\
\text { los hábitos de ejercicio } \\
\text { físico }\end{array}$ & $\begin{array}{l}\text { Incrementa con el nivel de } \\
\text { entrenamiento }\end{array}$ \\
\hline \multirow[t]{3}{*}{$\begin{array}{l}\text { Metodología de } \\
\text { estudio }\end{array}$} & Tamaño muestral & $\begin{array}{l}\text { Estudio con reducido } \\
\text { número de sujetos }\end{array}$ & $\begin{array}{l}\text { Disminuye la sensibilidad a } \\
\text { la detección de diferencias }\end{array}$ \\
\hline & $\begin{array}{l}\text { Número de } \\
\text { pruebas }\end{array}$ & $\begin{array}{l}\text { Estudios con una sola } \\
\text { prueba experimental }\end{array}$ & $\begin{array}{l}\text { Incrementa la variabilidad } \\
\text { en la respuesta al patrón y } \\
\text { al alimento o comida } \\
\text { probada }\end{array}$ \\
\hline & $\begin{array}{l}\text { Modelo } \\
\text { matemático ABC }\end{array}$ & $\begin{array}{l}\text { Modelos: ABC total, } \\
\text { incremental cortada, } \\
\text { ABC incremental neta, } \\
\text { etc. }\end{array}$ & $\begin{array}{l}\text { Incrementa la variabilidad } \\
\text { de resultados }\end{array}$ \\
\hline
\end{tabular}

ABC: Área bajo la curva. Modificado de Fernández, Miranda y Jiménez (2008)

De igual manera, las diferencias entre las medias ponderadas de 11 estudios con un máximo de 12 meses de observación, con dietas entre $40-60 \%$ de las calorías como glúcidos y estratificados con IG alto y bajo, demostraron que los sujetos que consumían dietas con IGs bajos tenían una reducción significativamente mayor de la hemoglobina glicosilada (8.0 a $7.2 \%)$ que la observada en los que consumían una dieta con IG alto [18]. Cuando se compararon dos cereales de desayuno con diferentes IGs (131 \pm 33 y $54.5 \pm 7.2$ ), la tasa de eliminación de glucosa fue una mayor determinante de la hiperglucemia postprandial. En este estudio se encontró que cereales de desayuno con menor IG indujeron hiperinsulinemia antes que los cereales con IG mayor, lo que resultó en un aumento en la eliminación de la glucosa sanguínea [14].

Otros estudios han relacionado el IG elevado con la resistencia insulínica (RI); sin embargo, en la actualidad se concluye que a pesar de haberse demostrado en algunas ocasiones asociación entre un elevado IG y RI y que la RI pudiera atenuarse al usar una dieta con bajo IG [30], no existen evidencias científicas calificadas para afirmar que el IG alto pueda inducir RI a largo plazo [18].

En cuanto a la prevención de la diabetes, algunos estudios epidemiológicos han asociado el IG y la CG elevados con el desarrollo de la diabetes mellitus tipo 2 (DMT2) [4]; sin embargo, algunos otros estudios no han encontrado asociación entre el IG o 
cuando las dietas son bajas en fibra dietética y altas en IG o CG [32].

Se han llevado a cabo varios estudios en personas con diabetes para examinar el efecto de dietas que difieren principalmente con respecto al IG sobre la hemoglobina glicosilada (HbA1c) y la fructosamina. Los datos de esos estudios forman las bases de dos meta-análisis. En uno de esos meta-análisis hubo una modesta reducción de la $\mathrm{HbA} 1 \mathrm{c}$ en personas que consumieron dietas con bajo IG, estimada en $0.33 \%(95 \%$ IC: $0.07,0.59)(22)$, mientras q en el otro la reducción fue de $0.27 \%(95 \%$ IC: $0.03,0.5)$ [33]. Las concentraciones de fructosamina fueron también menores en las intervenciones dietéticas con bajo IG.

Aunque un análisis del estudio Nurses' Health Study II encontró relación entre la CG y la diabetes gestacional [4], una revisión de estudios de la organización Cochrane concluyó que mientras que las dietas con bajo IG mostraban ser benéficas en algunos casos, los resultados de la revisión no fueron concluyentes [34], por lo que resulta particularmente importante la realización de más estudios.

Evidentemente, la utilidad del IG y la CG es más controversial en la prevención que en el control de la diabetes, sin embargo, en la práctica clínica y desde un punto de vista cualitativo, el IG y la CG como criterios para seleccionar los alimentos de la dieta de pacientes diabéticos son herramientas útiles que pueden evitar elevaciones y descensos súbitos de glucosa, indeseables en este padecimiento. Particularmente en el tratamiento de la diabetes, el uso adecuado del IG por parte del nutricionista para educar al paciente, complementado en los casos que lo requiera con otras herramientas como los equivalentes de glucosa o el índice insulinémico, ofrece muchas más ventajas que desventajas.

\subsubsection{Implicaciones del índice y carga glucémica en la presión arterial}

Según las investigaciones, con dietas bajas en IG y $C G$ se pueden alcanzar indirectamente mayores reducciones en la presión sanguínea que con planes de alimentación con IGs elevados mediante la reducción del peso corporal [35]; sin embargo, la efectividad de las dietas con bajo IG independientemente de la pérdida de peso es una variable que se debe seguir investigando. La dieta $\mathrm{DASH}$, que incluye productos relativamente bajos en IG, es la intervención más exitosa en el control de la hipertensión hasta el momento [36], además de que se ha demostrado que mejora la sensibilidad a la insulina [37].

\subsection{3 Índice glucémico y carga glucémica en el control y pérdida de peso}

Las dietas con un IG o CG bajos pueden ayudar en el control de peso influyendo en el apetito y en la "liberación sostenida de la energía" [12]. Una revisión centrada en la prevención del aumento de peso en la vida adulta sugirió que el consumo ad libitum de dietas bajas en grasa y altas en proteínas, aunado a la ingesta de carbohidratos complejos (teóricamente con menor IG) ayudó a prevenir el aumento de peso y que la selección de hidratos de carbono con un IG bajo podría mejorar el resultado [38]. Teniendo en cuenta que los alimentos con valores de IG alto son en gran medida alimentos basados en harinas o cereales, una dieta basada en alimentos con IGs medios y bajos, aunado a un adecuado control de las porciones ingeridas (es decir, con CG bajas y moderadas), es una estrategia saludable que permite llevar una dieta correcta, y, por ende, mantener el peso corporal. Sin embargo, se podría pensar que, si lo que se desea es perder peso y no mantenerlo, las dietas basadas en alimentos con IG y CG bajos y moderados pudieran no ser del todo eficaces, puesto que algunos de los sistemas dietéticos más efectivos diseñados para la pérdida de grasa corporal incluyen regímenes estrictos y un tanto desviados del concepto de dieta correcta. En este sentido, una revisión de artículos científicos de la fundación COCHRANE en torno al uso de IG y CG en sobrepeso y obesidad concluyó que las personas que siguieron dietas con bajo IG y CG perdieron más peso que los que tenían dietas con IG alto o incluso dietas hipocalóricas. También encontraron que las personas con consumo ad libitum de alimentos con bajo IG y CG perdieron tanto o más peso que las personas con dietas tradicionales para perder peso o hipolipídicas, argumentando que es más sencillo seguir un régimen dietético que incluya carbohidratos con IG y CG bajos que dejar de consumir carbohidratos como la mayoría de dietas efectivas de pérdida de peso 
sugieren [39]. De este modo, se puede sugerir que en individuos con sobrepeso y obesidad sin problemas de control glucémico que deseen perder peso, se pueden implementar dietas tradicionales de pérdida de peso o bien, dietas con bajos IG y CG, mientras que para personas con problemas de control glucémico es preferible implementar dietas basadas en el IG y CG en lugar de dietas tradicionales, pudiendo observar resultados tan favorables como los que muestran las dietas restrictivas convencionales.

\subsection{4 índice glucémico y carga glucémica en el desarrollo de cáncer}

Dentro de las publicaciones relevantes, una investigación que analizó la asociación del IG y la CG de la dieta con el riesgo de 19 tipos de cáncer de un estudio de casos y controles en población canadiense (NECSS), encontró que el IG y la CG estaban positivamente asociados con el cáncer de próstata y el cáncer colorrectal, respectivamente, pero no hubo asociaciones significativas entre IG, CG y cáncer de estómago, hígado, pulmón, mama, ovario, testículos, riñón, vejiga, cerebro, linfoma no Hodkin, leucemia y mieloma múltiple. Cuando los análisis de casos positivos de cáncer fueron estratificados por edad, sexo, IMC y consumo de tabaco, los riesgos tanto de cáncer de colon como rectal para la CG fueron aparentemente mayores en hombres. Por último, el riesgo de cáncer pancreático con respecto al IG fue aparentemente mayor en mujeres, y con respecto a la CG fue mayor en sujetos con sobrepeso y obesidad [40]. En un estudio de casos y controles se compararon 475 casos de mujeres mexicanas con cáncer de mama y se observó que aquellos con la mayor CG dietética, tenían un riesgo más elevado [41]. A pesar de algunos estudios como este que relacionan la CG con el riesgo de cáncer, los autores de una revisión hecha a un estudio epidemiológico prospectivo concluyeron que "No existe una relación consistente, fuerte $y$ estadísticamente significativa de las variables de la dieta y el cáncer de mama, incluyendo el IG y la CG [42]. Debemos hacer énfasis en que, aunque en padecimientos como este, el consumo de dietas con bajos IG CG es contradictorio y no parece tener en muchos casos una implicación directa, el cáncer es un padecimiento de etiología multifactorial y que cursar con sobrepeso y obesidad se ha correlacionado con el desarrollo de esta enfermedad en mayor medida, por lo que la utilidad combinada del IG y la CG para controlar la obesidad y así disminuir, por lo menos en cierta medida, el riesgo de cáncer, es bastante clara.

\subsection{5 Índice glucémico y carga glucémica en el deporte}

Aunque la ingesta de carbohidratos es de vital importancia para cubrir las demandas energéticas que genera el ejercicio [43], y consumir carbohidratos antes de un esfuerzo físico para aumentar el glucógeno muscular ha sido una práctica usada durante más de 25 años, existe controversia acerca de si el IG o la CG para el régimen de "carga energética" realmente importan [4]. La ciencia ha sugerido cambios en la utilización de los carbohidratos con respecto al momento de su implementación en las tres fases fisiológicas relacionadas con el ejercicio, a saber: antes del ejercicio, durante el ejercicio y después del ejercicio [44]. DeMarco Sucher, Cisar y Butterfield [45] investigaron el efecto de una comida de alto IG, una de bajo IG o únicamente agua en ciclistas, 30 minutos antes de pedalear dos horas a un $70 \%$ del VO2máx. Los autores reportaron un $59 \%$ en el tiempo de pedaleo antes de la fatiga y una reducción de la percepción del esfuerzo, lo cual se asoció a mayor glucemia durante el ejercicio después de la ingesta de la comida con bajo IG. Los autores de este estudio propusieron que los niveles de glucemia, al final del ejercicio de larga duración, se correlacionan negativamente con el IG de los alimentos ingeridos antes del esfuerzo [46]. La recomendación en este punto del ejercicio, de acuerdo con los resultados de este estudio, es que antes de realizar deportes de larga duración y de moderada y alta intensidad, se consuman alimentos con IGs bajos o alimentos con glucosa lentamente disponible, puesto que este tipo de alimentos aseguran una liberación lenta de carbohidratos que proporcionan energía a los músculos por mayor tiempo.

Pocos estudios han investigado el efecto de la ingestión continua de carbohidratos de alto o bajo IG sobre el rendimiento deportivo [44], no obstante, se sugiere que el alimento utilizado debe poseer alto IG 
para asegurar su rápida disponibilidad plasmática y mantener niveles estables de glucemia, lo que no se logra con los carbohidratos de bajo IG, que, dicho sea de paso, pudieran producir molestias gástricas; en contraste, la ingesta de carbohidratos de elevado IG en ejercicios de baja intensidad puede ocasionar el efecto contrario [8]. Esta recomendación basada en IG es general puesto que se debe considerar la duración e intensidad del deporte para recomendar alimentos con un determinado IG.

Los estudios demuestran que raciones con alto IG pueden recuperar más rápidamente los depósitos de glucógeno muscular y que los niveles de insulina generados por esta ración favorecen el transporte de glucosa al interior de la célula además de activar la enzima glucógena sintasa [8]. Se ha demostrado que la combinación de alimentos con IG elevado y proteína, posterior al esfuerzo, es la combinación ideal para lograr los mayores niveles de reposición glucogénica $[47,48]$.

Cabe destacar que el IG y la CG de la dieta pueden tener poco impacto para ciertos tipos de ejercicio o para las personas con diferentes niveles de formación deportiva. Por ejemplo, el IG de un desayuno consumido por mujeres antes de caminar a paso ligero no tuvo un impacto medible [49], y la CG e IG de la dieta de siete jugadores masculinos de fútbol semi-profesional proporcionada en una recuperación de 22 horas, no hicieron ninguna diferencia con respecto a la fatiga durante un evento o una carrera de ida y vuelta en cuanto al sprint y rendimiento en la distancia [50]. Aun así, el uso de estos parámetros en las recomendaciones dietéticas deportivas es, según nuestro criterio, útil en múltiples situaciones

\section{Consideraciones finales}

El IG y la CG son conceptos creados originalmente para el tratamiento de la diabetes tipo 1 que ofrecen una forma de clasificar los alimentos de acuerdo con el impacto en la glucemia plasmática, sin tomar en cuenta la sencillez o complejidad de los carbohidratos que contienen. Mientras que el IG únicamente compara la respuesta glucémica de un alimento cualquiera con un alimento control, la CG considera también la cantidad de carbohidratos totales disponibles.
De acuerdo a la literatura ambos conceptos tienen dos utilidades distintas, que ofrecen un control en la calidad de los alimentos por encima de la cantidad, analizando un alimento de manera independiente y no como parte de una comida mixta.

EI IG y la CG se pueden utilizar en la prevención de enfermedades, en el tratamiento de padecimientos crónicos, que ofrecen ventajas para el control de sobrepeso, obesidad y diabetes; sin embargo, necesitan el respaldo de otras herramientas para obtener los resultados esperados, que dependen de la experiencia del profesional de salud

\section{Referencias}

[1] Torres, N., Palacios-Gonzalez, B., Noriega-López, L., y Tovar-Palacio, A. R. (2006). Indice glicémico, índice insulinémico y carga glicémica de bebidas de soya con un contenido bajo y alto en hidratos de carbono. Revista de investigación clínica, 58(5), 487-497.

[2] Parada, J. A., y Rozowski, J. (2008). Relación entre la respuesta glicémica del almidón y su estado microestructural. Revista chilena de nutrición, 35(2), 84-92.

[3] Wolever, T. M. (2003). Carbohydrate and the regulation of blood glucose and metabolism. Nutrition reviews, 61(suppl_5), S40-S48.

[4] Jones, J. M. (2010). The role of glycemic index \& glycemic load on carbohydrate food quality: a status report. Wheat Foods Council.

[5] Mahan, L. K., Escott-Stump, S., y Raymond, J. L. (2013). Krause dietoterapia. Amsterdam: Elsevier.

[6] Livesey, G., Taylor, R., Livesey, H. F., Buyken, A. E., Jenkins, D. J., Augustin, L. S., ... y Willett, W. C. (2019). Dietary glycemic index and load and the risk of type 2 diabetes: Assessment of causal relations. Nutrients, 11(6), 1436.

[7] FAO. (1998). Carbohydrates in human nutrition, Reporto $f$ a Joint FAO/WHO Expert Consulation. FAO Food and Nutrition Paper 15, Roma. Consultado el 10 de mayo de 2020. http://www.fao.org/docrep/W8079E/W8079E00.htm

[8] Franco-Mijares, A. C., Cardona-Pimentel, G., Villegas-Canchola, K. P., Vázquez-Flores, A. L., Jáuregui-Vega, P. I., Jaramillo-Barrón, E., y Nava, A. (2013). Sobre el índice glucémico y el ejercicio físico en la nutrición humana. El Residente, 8(3), 89-96.

[9] Roberts, C. K., y Liu, S. (2009). Effects of glycemic load on metabolic health and type 2 diabetes mellitus. Journal of Diabetes Science and Technology, 3(4), 697-04.

[10] Gracey, M., Kretchmar, N., y Rossi, E. (1991). Sugars in nutrition. Lippincott Williams \& Wilkins.

[11] Ludwig, D. S. (2002). The glycemic index: physiological mechanisms relating to obesity, diabetes, and cardiovascular disease. Jama, 287(18), 2414-2423.

[12] Marsh, K., Barclay, A., Colagiuri, S., y Brand-Miller, J. (2011). Glycemic index and glycemic load of carbohydrates in the diabetes diet. Current diabetes reports, 11(2), 120-127. 
[13] Barclay, A. W., Petocz, P., McMillan-Price, J., Flood, V. M., Prvan, T., Mitchell, P., y Brand-Miller, J. C. (2008). Glycemic index, glycemic load, and chronic disease risk-a meta-analysis of observational studies. The American journal of clinical nutrition, 87(3), 627-637.

[14] Brand-Miller, J. C. (2004). Postprandial glycemia, glycemic index, and the prevention of type 2 diabetes. The American Journal of Clinical Nutrition, 80,243-244.

[15] Wahlqvist, M. L., Wilmshurst, E. G., Murton, C. R., y Richardson, E. N. (1978). The effect of chain length on glucose absorption and the related metabolic response. The American Journal of Clinical Nutrition, 31(11), 1998-2001.

[16] Lin, M. H. A., Wu, M. C., Lu, S., y Lin, J. (2010). Glycemic index, glycemic load and insulinemic index of Chinese starchy foods. World Journal of Gastroenterology: WJG, 16(39), 4973.

[17] Englyst, K. N., y Englyst, H. N. (2005). Carbohydrate bioavailability. British Journal of Nutrition, 94(1), 1-11.

[18] Arteaga, L. A. (2006). El índice glicémico: una controversia actual. Nutrición Hospitalaria, 21, 55-60.

[19] Salmerón, J., Ascherio, A., Rimm, E. B., Colditz, G. A., Spiegelman, D., Jenkins, D. J., ... y Willett, W. C. (1997). Dietary fiber, glycemic load, and risk of NIDDM in men. Diabetes care, 20(4), 545-550.

[20] Venn, B. J., y Green, T. J. (2007). Glycemic index and glycemic load: measurement issues and their effect on diet-disease relationships. European journal of clinical nutrition, 61(1), S122-S131.

[21] Brand-Miller, J., Hayne, S., Petocz, P., y Colagiuri, S. (2003). Lowglycemic index diets in the management of diabetes: a meta-analysis of randomized controlled trials. Diabetes care, 26(8), 2261-2267.

[22] Ebbeling, C. B., Leidig, M. M., Sinclair, K. B., Hangen, J. P., y Ludwig, D. S. (2003). A reduced-glycemic load diet in the treatment of adolescent obesity. Archives of pediatrics \& adolescent medicine, 157(8), 773-779.

[23] Noakes, M., Lau, C. W. H., Bowen, J., y Clifton, P. M. (2005). The effect of a low glycaemic index (GI) ingredient substituted for a high GI ingredient in two complete meals on blood glucose and insulin levels, satiety and energy intake in healthy lean women. Asia Pacific Journal of Clinical Nutrition, 14.

[24] Arcot, J., y Brand-Miller, J. (2005). A preliminary assessment of the glycemic index of honey. A Report for the Rural Industries Research and Development Corporation. Publication, (05/027).

[25] Torres-Maravilla, E., Blancas-Nápoles, J. A., Vázquez-Landaverde, P. A., Cristiani-Urbina, E., Mayorga-Reyes, L., \& Sánchez-Pardo, M. E. (2016). Evaluación de sedimentos de pulque (Xaxtle) como cultivo iniciador para obtener un producto de panificación de bajo índice glucémico. Agrociencia, 50(2), 183-200.

[26] Giacco, R., Parillo, M., Rivellese, A. A., Lasorella, G., Giacco, A., D'Episcopo, L., y Riccardi, G. (2000). Long-term dietary treatment with increased amounts of fiber-rich low-glycemic index natural foods improves blood glucose control and reduces the number of hypoglycemic events in type 1 diabetic patients. Diabetes care, 23(10), 1461-1466.

[27] Rodríguez, V. E. A., Rosendo, G. G., Murguía, N. A. R., Villanueva, J., y Sánchez, A. G. Q. G. (2013). Índice glucémico en alimentos compuestos. Rev Esp Nutr Comunitaria, 19(4), 216-223.

[28] Hernández, P., Mata, C., Lares, M., Velazco, Y., y Brito, S. (2013). Índice glicémico y carga glucémica de las dietas de adultos diabéticos y no diabéticos. Anales Venezolanos de Nutrición 26(1).
[29] Jiménez-Cruz, A., Seimandi-Mora, H., y Bacardi-Gascon, M. (2003). Efecto de dietas con bajo índice glucémico en hiperlipidémicos. Nutrición Hospitalaria, 18(6), 331-335.

[30] Daly, M. E., Vale, C., Walker, M., Alberti, K. G., y Mathers, J. C. (1997). Dietary carbohydrates and insulin sensitivity: a review of the evidence and clinical implications. The American journal of clinical nutrition, 66(5), 1072-1085.

[31] Mosdøl, A., Witte, D. R., Frost, G., Marmot, M. G., y Brunner, E. J. (2007). Dietary glycemic index and glycemic load are associated with highdensity-lipoprotein cholesterol at baseline but not with increased risk of diabetes in the Whitehall II study. The American journal of clinical nutrition, 86(4), 988-994.

[32] Riccardi, G., Rivellese, A. A., y Giacco, R. (2008). Role of glycemic index and glycemic load in the healthy state, in prediabetes, and in diabetes. The American journal of clinical nutrition, 87(1), 269S-274S.

[33] Opperman, A. M., Venter, C. S., Oosthuizen, W., Thompson, R. L., y Vorster, H. H. (2004). Meta-analysis of the health effects of using the glycaemic index in meal-planning. British Journal of Nutrition, 92(3), 367381.

[34] Vrolix, R., Van Meijl, L. E. C., y Mensink, R. P. (2008). The metabolic syndrome in relation with the glycemic index and the glycemic load. Physiology \& behavior, 94(2), 293-299

[35] Burton, P., y Lightowler, H. J. (2006). Influence of bread volume on glycaemic response and satiety. British Journal of Nutrition, 96(5), 877882.

[36] Johnston, C. S., y Buller, A. J. (2005). Vinegar and peanut products as complementary foods to reduce postprandial glycemia. Journal of the American Dietetic Association, 105(12), 1939-1942.

[37] Östman, E., Granfeldt, Y., Persson, L., y Björck, I. (2005). Vinegar supplementation lowers glucose and insulin responses and increases satiety after a bread meal in healthy subjects. European journal of clinical nutrition, 59(9), 983

[38] Astrup, A. (2001). Healthy lifestyles in Europe: prevention of obesity and type II diabetes by diet and physical activity. Public health nutrition, 4(2b), 499-515.

[39] Thomas, D., Elliott, E., y Baur, L. (2007). Dietas de bajo índice glucémico o baja carga glucémica para el sobrepeso y la obesidad. $L a$ biblioteca Cochrane Plus, 4.

[40] Hu, J., La Vecchia, C., Augustin, L. S., Negri, E., De Groh, M., Morrison, H., ... y Canadian Cancer Registries Epidemiology Research Group. (2013). Glycemic index, glycemic load and cancer risk. Annals of oncology, 24(1), 245-251.

[41] Lajous, M., Willett, W., Lazcano-Ponce, E., Sanchez-Zamorano, L. M., Hernandez-Avila, M., y Romieu, I. (2005). Glycemic load, glycemic index, and the risk of breast cancer among Mexican women. Cancer Causes \& Control, 16(10), 1165-1169.

[42] Michels, K. B., Mohllajee, A. P., Roset-Bahmanyar, E., Beehler, G. P., y Moysich, K. B. (2007). Diet and breast cancer: a review of the prospective observational studies. Cancer: Interdisciplinary International Journal of the American Cancer Society, 109, 2712-2749.

[43] Borer, K. T. (2003). Hormonal regulation of fuel use in exercise. Exercise endocrinology, 97-120.

[44] Fernández, J. M., Miranda, J. L., y Jiménez, F. P. (2008). Índice glucémico y ejercicio físico. Revista Andaluza de Medicina del Deporte, 1(3), 116-124. 
[45] DeMarco, H. M., Sucher, K. P., Cisar, C. J., y Butterfield, G. E. (1997). Preexercise Carbohydrate Meals: Application of Glycemic Index. Journal of the American Dietetic Association, 97(9), A14.

[46] Thomas, D. E., Brotherhood, J. R., y Brand, J. C. (1991). Carbohydrate feeding before exercise: effect of glycemic index. International journal of sports medicine, 12(02), 180-186.

[47] Aoi, W., Naito, Y., y Yoshikawa, T. (2006). Exercise and functional foods. Nutrition journal, 5(1), 15 .

[48] Mondazzi, L., y Arcelli, E. (2009). Glycemic index in sport nutrition. Journal of the American College of Nutrition, 28(sup4), 455S463S.

[49] Backhouse, S. H., Williams, C., Stevenson, E., y Nute, M. (2007) Effects of the glycemic index of breakfast on metabolic responses to brisk walking in females. European journal of clinical nutrition, 61(5), 590-596.

[50] Erith, S., Williams, C., Stevenson, E., Chamberlain, S., Crews, P., y Rushbury, I. (2006). The effect of high carbohydrate meals with different glycemic indices on recovery of performance during prolonged intermittent high-intensity shuttle running. International journal of sport nutrition and exercise metabolism, 16(4), 393-404. 